\title{
Clinical comparative analysis of histidine-tryptophan-ketoglutarate solution and St. Thomas crystalloid cardioplegia: A 12-year study from a single institution
}

\author{
YING-ZHONG LIN $^{1 *}$, JING-BIN HUANG $^{1,2^{*}}$, XIANG-WEI LI ${ }^{1}$, XIAN-MING TANG ${ }^{1}$, \\ WEI-JUN LU ${ }^{1}$, ZHAO-KE WEN ${ }^{1}$, JIAN LIANG ${ }^{2}$, DIAN-YUAN LI ${ }^{3}$ and HAO WANG ${ }^{1}$
}

${ }^{1}$ Department of Cardiothoracic Surgery, People's Hospital of Guangxi Zhuang Autonomous Region, Nanning, Guangxi 530021; ${ }^{2}$ Department of Cardiothoracic Surgery, Ruikang Hospital Affiliated to Guangxi University of Chinese Medicine, Nanning, Guangxi 530011; ${ }^{3}$ Pediatric Center of Cardiac Surgery, Cardiovascular Institute and Fuwai Hospital, Chinese Academy of Medical Sciences and Peking Union Medical College, Beijing 100037, P.R. China

Received October 26, 2015; Accepted November 18, 2016

DOI: $10.3892 / \mathrm{etm} .2017 .4814$

\begin{abstract}
Cardioplegic reperfusion during a long-term ischemic period interrupts cardiac surgery and increases cellularedemadue to repeated administration. The present clinical study compared the protective effects of histidine-ketoglutarate-tryptophan (HTK) solution and St. Thomas crystalloid cardioplegia. Clinical experiences of the myocardial protection induced by one single perfusion with HTK were reviewed in high-risk patients with severe pulmonary arterial hypertension associated with complex congenital heart disease. This retrospective study included 88 high-risk patients (aortic cross-clamp time, $>120$ min) between March 2001 and July 2012. The cohort was divided into two groups according to the technique used. Either myocardial protection was performed with one single perfusion with HTK solution (HTK group) or with conventional St. Thomas crystalloid cardioplegia (St group). The duration of cardiopulmonary bypass did not differ between the two groups. The mortality, morbidity, intensive care unit (ICU) stay, postoperative hospitalization, and transfusions of HTK group are significantly lower than those of the St group $(\mathrm{P}<0.05)$. Univariate and multivariate analysis demonstrated that HTK is a statistically significant independent predictor of decreased early mortality and morbidity $(\mathrm{P}<0.05)$. In conclusion, the present findings suggested that HTK solution decreases mortality, morbidity, ICU stay, postoperative hospitalization,
\end{abstract}

Correspondence to: Dr Jing-Bin Huang, Department of Cardiothoracic Surgery, People's Hospital of Guangxi Zhuang Autonomous Region, 6 Taoyuan Road, Nanning, Guangxi 530021, P.R. China

E-mail: b2007117@hotmail.com

${ }^{*}$ Contributed equally

Key words: histidine-tryptophan-ketoglutarate, myocardial protection, cardioplegia, cardiac surgery and transfusions in high-risk patients with severe pulmonary arterial hypertension associated with complex congenital heart disease.

\section{Introduction}

With the development of cardiac surgery, an increasing number of high-risk patients with complex congenital heart disease have undergone cardiac surgery $(1,2)$. Ischemia-reperfusion injury during cardiac surgery is associated with increased mortality and morbidity. The improvement of myocardium tolerance to ischemia for high-risk patients with complex congenital heart disease is a key issue (3). However, the optimal myocardial protection strategy for high-risk patients with complex congenital heart disease remains controversial $(1,4)$. There are two main groups of cardioplegic solutions; one is based on extracellular components with high potassium, magnesium and bicarbonate levels and the other is based on intracellular electrolytes. The histidine-ketoglutarate-tryptophan (HTK) solution, which is based on intracellular electrolytes, was introduced by Preusse et al in 1981 (5). A single-dose strategy for myocardial protection, which avoids interrupting the procedure to re-administer cardioplegia, is preferable during lengthy surgery.

Histidine, tryptophane and ketoglutarate are the components of HTK solution. Histidine has a buffer effect and may enhance the efficiency of anaerobic glycolysis, tryptophane stabilizes the cell membrane and the addition of mannitol decreases cellular oedema, whereas ketoglutarate is a precursor of nicotinamide adenine dinucleotide (6). Several studies have demonstrated the efficacy of the HTK solution based on biochemical markers or physiological evaluation in experimental models (7-9). HTK solution helps to preserve myocardial adenosine triphosphate stores, improve post-arrest contractile function and minimize myocardial necrosis $(10,11)$. HTK solution has also been shown to preserve the coronary artery endothelium, which may help to improve functional cardiac recovery $(12,13)$. Careaga et al (14) reported that patients treated with HTK solution had a lower incidence of arrhythmias, length-of-stay in the intensive care unit (ICU) and inotropic support, postoperatively. 
It was hypothesized that a single-dose of HTK may provide superior myocardial protection than cold crystalloid cardioplegia in high-risk patients. The present clinical study compared the protective effects of HTK Solution and St. Thomas crystalloid cardioplegia in high-risk patients with severe pulmonary arterial hypertension associated with complex congenital heart disease.

\section{Materials and methods}

Patients. Cardiac catheterization data was collected from patients under general anesthesia. Severe pulmonary arterial hypertension was defined as mean pulmonary pressure $>50 \mathrm{mmHg}$ or systolic pulmonary/systemic pressure ratio $>0.8$. A total of 88 high-risk patients (47 males, 48 females; aortic cross-clamp time, $>120 \mathrm{~min})$ aged $>6$ months $(3.17 \pm 3.36$ years $)$ with dextro-transposition of the great arteries (d-TGA) and nonrestrictive ventricular septal defect or Taussig-Bing anomaly and severe pulmonary arterial hypertension who underwent arterial switch surgery (two ventricles repair) at Fuwai Hospital between March 2001 and July 2012 were included in the study. Patients were divided into two groups: HTK group (myocardial protection was performed with a single perfusion of HTK solution) and the St group (control group; myocardial protection with conventional St. Thomas cold potassium cardioplegia) (Table I). Inclusion criteria included: Patients aged $>6$ months who were diagnosed as d-TGA and nonrestrictive ventricular septal defect or Taussig-Bing anomaly and severe pulmonary arterial hypertension. Exclusion criteria included: Patients aged $<6$ months; mean pulmonary arterial pressure $<50 \mathrm{mmHg}$ or systolic pulmonary/systemic pressure ratio $<0.8$; or 21 trisomy. Diagnosis (according to the International Congenital Heart Surgery Nomenclature) was made on the basis of echocardiographic and cardiac angiographic findings and was confirmed during surgery. Hospital charts, echocardiographic and cardiac catheterization data and operative reports were reviewed. Cardiac catheterization was performed under general anesthesia. Preoperative pulmonary artery pressure and pulmonary vascular resistance were measured according to the conventional cardiac catheterization protocol and Fick method $(15,16)$ The Medical Ethics Committee of Fuwai Cardiovascular Disease Hospital approved the study protocol, and approval was granted to waive the requirement for patient consent for publishing follow-up data about the present patients.

Data collection. Clinical records of 88 high-risk patients included in the present study were retrospectively reviewed. If a patient succumbed to their symptoms, their death certificate and the medical records of the hospital and physician were reviewed. In the present study, early mortality was defined as mortality prior to hospital discharge or within 30 days of arterial switch surgery.

Anesthesia management. Prior to surgery, anesthesia was induced with intravenous ketamine $(1.5 \mathrm{mg} / \mathrm{kg}$; Xi'an HanFeng Pharmaceutical Co. Ltd., Xi'an, China), fentanyl (30 $\mu \mathrm{g} / \mathrm{kg}$; Yichang Humanwell Pharmaceutical Co. Ltd., Yichang, China), and pancuronium $(0.15 \mathrm{mg} / \mathrm{k}$; Organon International, Oss, Netherlands). During surgery, fentanyl $(0.3 \mu \mathrm{g} \cdot \mathrm{kg}-1 \cdot \mathrm{min}-1$; Yichang Humanwell Pharmaceutical Co. Ltd.) was used for
Table I. Compositions of the two cardioplegic solutions.

\begin{tabular}{lcc}
\hline Substrate $(\mathrm{mM})$ & HTK $^{\mathrm{a}}$ & St. Thomas $^{\mathrm{b}}$ \\
\hline $\mathrm{K}^{+}$ & 10 & 16 \\
$\mathrm{Na}^{+}$ & 15 & 116 \\
$\mathrm{Ca}^{2+}$ & 0.02 & 1.2 \\
$\mathrm{Mg}^{2+}$ & 4 & 16 \\
Sodium bicarbonate & 0 & 10 \\
Histidine & 180 & 0 \\
Tryptophan & 2 & 0 \\
$\alpha$-ketoglutarate & 1 & 0 \\
Mannitol & 30 & 0 \\
Theoric osmolality (mOsm/l) & 310 & 318 \\
\hline
\end{tabular}

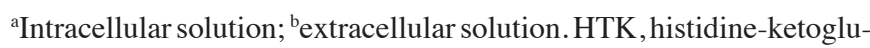
tarate-tryptophan; St. Thomas, St. Thomas crystalloid cardioplegia.

maintenance. An arterial line in the radial artery or femoral artery was used for hemodynamic monitoring. Specifically, a central venous pressure catheter was installed via the subclavian vein, and a urinary catheter was installed.

Cardiopulmonary bypass. Cardiopulmonary bypass was performed using a Dideco 901 or 902 hollow fiber oxygenator (Sorin Group, Mirandola, Italy), and a roller pump (Jostra $\mathrm{GmbH}$, Munich, Germany) with non-pulsatile flow. A tubing pack, which contained a crystalloid cardioplegia delivery system (Beijing Perfect Chemical Material Co. Ltd., Beijing, China) and an arterial clotting time was maintained above $400 \mathrm{sec}$ during cardiopulmonary bypass. Heparin (Shanghai No. 1 Biochemical Pharmaceutical Co., Ltd., Shanghai, China) was neutralized with protamine chloride $(4 \mathrm{mg} / \mathrm{kg}$ ) after cardiopulmonary bypass.

Infusion of cardioplegia. Under cardiopulmonary bypass and aortic cross-clamping, cardioplegic arrest was induced. HTK solution $\left(4-8^{\circ} \mathrm{C}\right)$ was perfused as a single dose $(40-50 \mathrm{ml} / \mathrm{kg})$ via the aortic root at an initial perfusion pressure of $80-100 \mathrm{mmHg}$. Infusion was maintained at $40-60 \mathrm{mmHg}$ over 5-7 min. St. Thomas solution $\left(4-8^{\circ} \mathrm{C}\right.$; initial dose, $20 \mathrm{ml} / \mathrm{kg}$; maintenance dose, $10 \mathrm{ml} / \mathrm{kg}$ ) was perfused (antegrade) every $30 \mathrm{~min}$ at a pressure of 100-120 mmHg. Cardiopulmonary bypass time and aortic cross-clamping time were recorded. The presence of arrhythmias during reperfusion after aortic cross-clamping and during the postoperative period was evaluated.

Surgical technique. Median sternotomy and hypothermic cardiopulmonary bypass with ultrafiltration technique were routinely used. Cardioplegia solution was administered (HTK solution: 40-50 ml/kg; St. Thomas solution: Initial dose $20 \mathrm{ml} / \mathrm{kg}$, maintenance dose $10 \mathrm{ml} / \mathrm{kg}$ ). Surgery was performed on cardiopulmonary bypass at low flow $(50 \mathrm{ml} / \mathrm{kg} / \mathrm{min})$ with a rectal temperature of $18-22^{\circ} \mathrm{C}$. Ventricular and atrial septal defect and patent ductus arteriosus were completely closed. The aorta was transected and the aortic root was fully dissected. A large aortic button containing the coronary orifice was harvested. The pulmonary 
Table II. Demographic and operative data.

\begin{tabular}{lccc}
\hline Variable & $\begin{array}{c}\text { HTK group } \\
(\mathrm{n}=70)\end{array}$ & $\begin{array}{c}\text { St group } \\
(\mathrm{n}=18)\end{array}$ & P-value \\
\hline Patients, $\mathrm{n}$ & 70 & 18 & \\
Male, $\mathrm{n}(\%)$ & 35 & 12 & 0.833 \\
Age, years & $3.4 \pm 0.4$ & $2.2 \pm 0.5$ & 0.176 \\
Weight, kg & $12.0 \pm 0.9$ & $10.2 \pm 1.2$ & 0.347 \\
c/t ratio & $0.6 \pm 0.01$ & $0.6 \pm 0.01$ & 0.614 \\
CPB time, min & $239.9 \pm 6.8$ & $233.4 \pm 11.4$ & 0.654 \\
Aortic cross-clamp & $170.8 \pm 4.2$ & $158.8 \pm 5.8$ & 0.181 \\
time, min & & & \\
SPO 2 & $74.9 \pm 1.9$ & $73.4 \pm 3.2$ & 0.719 \\
Pre-op mPAP & $65.3 \pm 1.6$ & $61.2 \pm 3.1$ & 0.246 \\
Post-op mPAP & $31.9 \pm 1.3$ & $31.8 \pm 2.2$ & 0.965 \\
Pre-op PVR & $524.4 \pm 134.9$ & $439.4 \pm 120.3$ & 0.769 \\
Ultrafiltration, ml & $831.9 \pm 148.7$ & $545.6 \pm 62.4$ & 0.336 \\
Chest drainage, ml & $401.6 \pm 89.2$ & $367.1 \pm 71.5$ & 0.848 \\
\hline
\end{tabular}

Data are presented as the mean \pm standard error of the mean, unless otherwise stated. HTK, histidine-ketoglutarate-tryptophan solution; St, St. Thomas crystalloid cardioplegia; c/t ratio, cardiothoracic ratio; CPB time, time of cardiopulmonary bypass; SPO2, pulse oxygen saturation; Pre-op mPAP, preoperative mean pulmonary arterial pressure; Post-op mPAP, postoperative mean pulmonary arterial pressure; Pre-op PVR, preoperative pulmonary vascular resistance.

trunk was transected, pulmonary branches dissected, and the Lecompte maneuver (17) was performed. The coronary artery was reimplanted to an appropriate site of the neo-aorta. Pulmonary artery reconstruction was performed using a fresh autologous pantaloon-shaped pericardial patch. Associated anomalies were corrected simultaneously. Associated anomalies include patent ductus arteriosus, atrial septal defect, mild left ventricular outflow tract obstruction, mild right ventricular outflow tract obstruction, mitral insufficiency, tricuspid insufficiency, pulmonary veinstenosis, aortic arch coarctation, major aortopulmonary collaterals and anomalous origin of the right pulmonary artery from the ascending aorta. Postoperative pulmonary artery pressure measurements were recorded in the operating room at the end of the surgery.

Follow-up. All survivors were followed-up to the end date of the study (July 2012). All patients at the outpatient department were subjected to electrocardiogram, X-ray chest film and echocardiogram analysis. Patients were followed-up at the outpatient department once every three months. At the final follow-up, patients were contacted by telephone or were directly interviewed at the outpatient department.

Statistical analysis. All analyses were performed using SPSS version 18.0 software (SPSS Inc., Chicago, IL, USA). Continuous variables were expressed as mean \pm standard error of the mean and were compared by a two-tailed Student's t-test. Survival rates were estimated using the Kaplan-Meier method. Comparison of multiple mean values was performed by analysis of variance. Discrete variables were expressed as percentages and compared
Table III. Hospital mortality and morbidity.

\begin{tabular}{|c|c|c|c|}
\hline Variable & $\begin{array}{l}\text { HTK group, } \\
n=70 . n(\%)\end{array}$ & $\begin{array}{c}\text { St group, } \\
\mathrm{n}=18 . \mathrm{n}(\%)\end{array}$ & P-value \\
\hline Mortality & $2(2.8)$ & $4(22.2)$ & 0.015 \\
\hline \multicolumn{4}{|l|}{ Cause } \\
\hline Severe arrhythmia & $1(1.4)$ & $2(11.1)$ & 0.105 \\
\hline $\mathrm{PH}$ crisis & $1(1.4)$ & 0 & 1.000 \\
\hline MOF & 0 & $2(11.1)$ & 0.040 \\
\hline Morbidity & $14(20)$ & $12(66.7)$ & $<0.001$ \\
\hline \multicolumn{4}{|l|}{ Cause } \\
\hline $\mathrm{PH}$ crisis & $1(1.4)$ & 0 & 1.000 \\
\hline LOS & $1(1.4)$ & $1(5.6)$ & 0.369 \\
\hline Arrhythmia & 0 & $1(5.6)$ & 0.205 \\
\hline Respiratory failure & 0 & $1(5.6)$ & 0.205 \\
\hline Pneumonia & $5(7.1)$ & $1(5.6)$ & 1.000 \\
\hline Pneumothorax & 0 & $1(5.6)$ & 0.205 \\
\hline pleural effusion & $5(7.1)$ & $1(5.6)$ & 1.000 \\
\hline Hemorrhage & $2(2.9)$ & $2(11.1)$ & 0.184 \\
\hline Sepsis & 0 & $1(5.6)$ & 0.205 \\
\hline Gastrointestinal & 0 & $1(5.6)$ & 0.205 \\
\hline MOF & 0 & $2(11.1)$ & 0.040 \\
\hline
\end{tabular}

HTK, histidine-ketoglutarate-tryptophan solution; St, St. Thomas crystalloid cardioplegia; PH, pulmonary arterial hypertension; LOS, low-output syndrome; MOF, multiple organ failure.

Table IV. Resource utilization.

\begin{tabular}{lccc}
\hline Variable & $\begin{array}{c}\text { HTK group } \\
(\mathrm{n}=70)\end{array}$ & $\begin{array}{c}\text { St group } \\
(\mathrm{n}=18)\end{array}$ & P-value \\
\hline Ventilation, h & $112.6 \pm 29.6$ & $219.2 \pm 61.8$ & 0.118 \\
ICU stay, days & $10.5 \pm 1.4$ & $20.1 \pm 4.4$ & 0.009 \\
Post-op hospitalization, & $19.1 \pm 1.5$ & $26.1 \pm 4.3$ & 0.063 \\
days & & & \\
Transfusions, ml & $772.1 \pm 88.7$ & $1352.9 \pm 214.7$ & 0.007 \\
\hline
\end{tabular}

Data are presented as the mean \pm standard error of the mean. HTK, histidine-ketoglutarate-tryptophan solution; St, St. Thomas crystalloid cardioplegia; ICU, intensive care unit; Post-op hospitalization, postoperative hospitalization.

by Fisher's exact test or Pearson's $\chi^{2}$ test, as required. End-points of the study included prevalence and cause of hospital mortality, prevalence and cause of hospital morbidity, resource utilization (assisted ventilation, intensive care and blood transfusions) and postoperative hospital stay. Associations with perioperative risk factors were assessed by means of contingency table methods and logistic regression analysis. To explore the simultaneous effects of perioperative characteristics on early mortality, variables that were significant at the 0.1 level in univariate analysis were included in a multivariate logistic regression model. $\mathrm{P}<0.05$ was considered to indicate a statistically significant difference. 
Table V. Alterations in serum sodium in the histidine-ketoglutarate-tryptophan group.

\begin{tabular}{|c|c|c|c|c|c|}
\hline Variable & Prior to $\mathrm{CPB}$ & After clamping & After declamping & After CPB & In PICU \\
\hline $\operatorname{Hct}(\%)$ & $37 \pm 3$ & $26 \pm 4$ & $29 \pm 1$ & $34 \pm 3$ & $35 \pm 5$ \\
\hline $\mathrm{Na}^{+}(\mathrm{mmol} / \mathrm{l})$ & $136.8+2.5$ & $132.6 \pm 3.1^{\mathrm{a}}$ & $134.6 \pm 2.1$ & $137.6 \pm 2.3$ & $139.8 \pm 2.1$ \\
\hline $\mathrm{Lac}(\mathrm{mmol} / \mathrm{l})$ & $0.9 \pm 0.2$ & $1.5 \pm 0.3$ & $2.1 \pm 1.5$ & $2.3 \pm 1.6$ & $1.8 \pm 1.3$ \\
\hline Osm (mOsm/kg) & $272.8 \pm 4.3$ & $266.8 \pm 7.3$ & $272.6 \pm 5.1$ & $277.8 \pm 5.1$ & $282.8 \pm 5.3$ \\
\hline
\end{tabular}

Data are presented as the mean \pm standard error of the mean. CPB, cardiopulmonary bypass; Hct, hematocrits; Lac, lactate; Osm, osmotic pressure; PICU, pediatric intensive care unit. ${ }^{a} \mathrm{P}<0.001$.

Table VI. Spontaneous defibrillation.

\begin{tabular}{lccc}
\hline Group & $\mathrm{n}$ & $\begin{array}{c}\text { Spontaneous } \\
\text { defibrillation, } \mathrm{n}(\%)\end{array}$ & P-value \\
\hline HTK & 70 & $65(92.9)$ & 0.027 \\
St & 18 & $13(72.2)$ & \\
\hline
\end{tabular}

HTK, histidine-ketoglutarate-tryptophan solution; St, St. Thomas crystalloid cardioplegia;.

\section{Results}

Patients. Table II shows the demographic and operative data. Preoperative cardiac catheterization data were available for all patients. No significant differences were observed between any of the variables.

Mortality and morbidity. The mortality rate during surgery was 6/88. Mortality in the HTK group was significantly lower than that of the St group ( 2.8 vs. $22.2 \%$; $\mathrm{P}=0.015)$, suggesting that HTK solution may decrease mortality. Multiple organ failure in HTK group was significantly lower than that in St group ( 0.0 vs. $11.1 \%$; $\mathrm{P}=0.040)$, suggesting that the effect of organ preservasion of HTK solution is better than that of St. Thomas crystalloid cardioplegia. The incidence of morbidity in HTK group was significantly lower in than that in St group (20 vs. 66.7\%; P<0.001; Table III), suggesting that HTK solution has better clinical effect than St. Thomas crystalloid cardioplegia.

Resource utilization. In the HTK group, the duration of ICU stay was significantly shorter than that of the St group (10.5 \pm 1.4 vs. $20.1 \pm 4.4$ days; $\mathrm{P}=0.009)$, suggesting that the clinical effect of HTK solution is better than that of St. Thomas crystalloid cardioplegia. The mean volume of transfusions for patients was significantly reduced in the HTK group $(772.1 \pm 88.7$ vs. $1352.9 \pm 214.7 \mathrm{ml}$; $\mathrm{P}=0.007$; Table IV); however, the reason for this remains to be elucidated.

Alterations in serum sodium in HTK group. Table V shows the changes of serum sodium observed in the HTK group. The results indicated that serum sodium in the HTK group decreased after clamping $(\mathrm{P}<0.001$; Table V).
Spontaneous defibrillation. Following reperfusion, rates of spontaneous defibrillation in the HTK group were significantly higher than those of the St group (92.9 vs. 72.2\%; $\mathrm{P}=0.027$ ) (Table VI), indicating that the myocardial protection effect of HTK solution is superior to that of St. Thomas crystalloid cardioplegia.

Follow-up results. Follow-up was successfully completed by $90.2 \%(74 / 82)$ of patients. The mean duration of follow-up was $58.8 \pm 26.8$ months; two late deaths (deaths that occurred $>2$ months post-surgery) occurred due to sudden death with no known reason. The overall survival rate of patients in the present study was $97.3 \%$ (72/74). The latest follow-up data showed that $2.8 \%$ of survivors were in New York Heart Association Class II and $97.2 \%$ were in Class I, showing that the midterm results of the operation are excellent.

Analysis of risk factors associated with early mortality and morbidity. Both univariate and multivariate analysis showed that HTK was associated with decreased early mortality [odds ratio $(\mathrm{OR}=0.103, \mathrm{P}=0.013$ and $\mathrm{OR}=0.135, \mathrm{P}=0.045$, respectively) and morbidity $(\mathrm{OR}=0.132, \mathrm{P}<0.001$ and $\mathrm{OR}=0.097, \mathrm{P}<0.001$, respectively; Table VII). This strongly indicates that the myocardial protection effect of HTK solution is superior to that of St. Thomas crystalloid cardioplegia.

\section{Discussion}

Mortality and morbidity rates for high-risk patients with complex congenital heart disease are relatively high; therefore, although it remains difficult, the optimal myocardial protection strategy for high-risk patients should be urgently determined $(6,18)$. Data collected from instances in which HTK solution has been used for high-risk patients with complex congenital heart disease remains rare (19-22).

A study of Fuwai Hospital compared the myocardial protective effect induced by HTK solution and conventional St. Thomas crystalloid cardioplegia on the long-term ischemic period (cross-clamping time, $>90 \mathrm{~min}$ ) during severe complex pediatric cardiac surgery without pulmonary arterial hypertension. The results demonstrated that mortality rates and the levels of CK in the HTK solution group were significantly lower, as compared with these values in the St. Thomas crystalloid cardioplegia group $(\mathrm{P}<0.05)$. Patients with severe pulmonary arterial hypertension associated with congenital heart disease are difficult to treat in clinic practice. Older patients 
Table VII. Analysis of RF for early mortality and morbidity.

\begin{tabular}{|c|c|c|c|}
\hline Model & OR & $95 \% \mathrm{CI}$ & P-value \\
\hline \multicolumn{4}{|c|}{ Univariate analysis of risk factors for mortality } \\
\hline HTK & 0.103 & $0.017-0.618$ & 0.013 \\
\hline \multicolumn{4}{|c|}{ Multivariate analysis of risk factors for mortality } \\
\hline HTK & 0.135 & $0.019-0.954$ & 0.045 \\
\hline Sex & 2.389 & $0.338-16.867$ & 0.383 \\
\hline Age & 0.574 & $0.558-1.382$ & 0.668 \\
\hline \multicolumn{4}{|c|}{ Univariate analysis of risk factors for morbidity } \\
\hline HTK & 0.132 & $0.042-0.409$ & 0.000 \\
\hline \multicolumn{4}{|c|}{ Multivariate analysis of risk factors for morbidity } \\
\hline HTK & 0.097 & $0.028-0.336$ & 0.000 \\
\hline Sex & 0.553 & $0.179-1.706$ & 0.303 \\
\hline Age & 1.208 & $1.037-1.407$ & 0.015 \\
\hline
\end{tabular}

OR, odds ratio; CI, confidence interval; UVA, univariate analysis; multivariate analysis; RF, risk factors; HTK, histidine-ketoglutarate-tryptophan solution.

(aged >6 months) with complex congenital heart disease with severe pulmonary arterial hypertension are at a higher risk of developing postoperative pulmonary arterial hypertension (23). Postoperative pulmonary arterial hypertension is a major determinant of perioperative morbidity and mortality $(24,25)$.

Conventional St. Thomas cold potassium cardioplegia solution is routinely used at Fuwai Hospital, and HTK solution is used in patients with complex congenital heart disease. A comparison of the myocardial protection of a single dose of HTK solution and St. Thomas crystalloid cardioplegia in high-risk patients with severe pulmonary arterial hypertension associated with complex congenital heart disease may have more power to detect significant differences in myocardial protection between HTK solution and St. Thomas crystalloid cardioplegia. Older patients (aged $>6$ months) with complex congenital heart disease with severe pulmonary arterial hypertension were included in the present study, whereas patients without severe pulmonary arterial hypertension were excluded.

In the present 12-year retrospective analysis of Fuwai Hospital, the mortality rate and incidence of morbidity in the HTK group was significantly lower than that of the St group, and the ICU stays of patients in the HTK group were significantly shorter than those in the St group. The mean volume of transfusions for patients was significantly less in the HTK group, as compared with the St group. Following reperfusion, rates of spontaneous defibrillation in the HTK group were significantly higher than those observed in the St group. Univariate and multivariate analyses showed that HTK was associated with decreased early mortality and morbidity.

The present study demonstrated that HTK solution is more effective than the St. Thomas crystalloid cardioplegia at protecting the myocardium from ischemia. However, subsequent prospective randomized controlled trials in a uniform group of high-risk patients are required to detect equivalence or important differences.

Blood cardioplegia has typically been used as the standard to protect the myocardium. Conventional St. Thomas crystalloid cardioplegia and blood cardioplegia must be repeated every 20-30 min and the surgical procedure must be suspended during infusion. An uninterrupted surgical field is desirable, and single-dose cardioplegia may be preferable in more complex cardiac procedures to avoid disturbing the technical flow of the surgery. Del Nido cardioplegia was formulated to act as single-dose administration in pediatric patients (26,27). Del Nido cardioplegia has not been widely used in Fuwai Hospital.

HTK induces fluctuations in sodium concentration, thus particular attention should be paid to low $\mathrm{Na}^{+}$levels. Hyponatremia becomes deleterious if osmolarity simultaneously becomes hypoosmolar. Aspirating HTK solution into a blood cell saver as much as possible avoids large volumes of HTK solution entering the cardiopulmonary bypass circuit, which would otherwise induce severe hyponatremia. Clinicians must avoid overtreatment of low sodium content to prevent severe neurologic complications. The application of small volumes $(25-100 \mathrm{ml})$ of $\mathrm{NaCl}(5.85 \%)$ or $\mathrm{NaHCO}_{3}(8.4 \%)$ to the circuit is recommended in adults during cardioplegic delivery to counteract low blood sodium content (28).

In conclusion, the present study showed that HTK solution decreases mortality, morbidity, ICU stay, postoperative hospitalization, and transfusions in high-risk patients with complex congenital heart disease. A prospective randomized controlled trial is required to determine the superior myocardial protective effects of HTK solution, as compared with conventional St. Thomas crystalloid cardioplegia.

\section{Acknowledgments}

This work was supported by the Natural Science Foundation of China (grant no. 81360014), the Natural Science Foundation of Guangxi (grant no. 2014GXNSFAA118234), the Guangxi Key Scientific and Technological Project (grant no. 2013BC26236) and the Guangxi Health Department Projects (grant no. GZPT13-27). 


\section{References}

1. Huang JB, Liu YL, Yu CT, Lv XD, Du M, Wang Q and Kong B: Lung biopsy findings in previously inoperable patients with severe pulmonary arterial hypertension associated with congenital heart disease. Int J Cardiol 151: 76-83, 2011.

2. Huang JB, Liang J and Zhou LY: Eisenmenger Syndrome: Not always inoperable. Respir Care 57: 1488-1495, 2012.

3. Liu J, Feng Z, Zhao J, Li B and Long C: The myocardial protection of HTK cardioplegic solution on the long-term ischemic period in pediatric heartsurgery. ASAIO J 54: 470-473, 2008.

4. Viana FF, Shi WY, Hayward PA, Larobina ME, Liskaser F and Matalanis G: Custodiol versus blood cardioplegia in complex cardiac operations: An Australian experience. Eur J Cardiothorac Surg 43: 526-531, 2013.

5. Preusse CJ, Gebhard MM and Bretschneider HJ. Myocardial "equilibration processes" and myocardial energy turnover during initiation of artificial cardiac arrest with cardioplegic solution - reasons for a sufficiently long cardioplegic perfusion. Thorac Cardiovasc Surg 29: 71-76, 1981.

6. Braathen B, Jeppsson A, Scherstén H, Hagen OM, Vengen $\varnothing$, Rexius H, Lepore V and Tønnessen T: One single dose of histidine-tryptophan-ketoglutarate solution gives equally good myocardial protection in elective mitral valve surgery as repetitive cold blood cardioplegia: A prospective randomized study. J Thorac Cardiovasc Surg 141: 995-1001, 2011.

7. Chen Y, Liu J, Li S, Li W, Yan F, Sun P, Wang H and Long C: Which is the better option during neonatal cardiopulmonary bypass: HTK solution or cold blood cardioplegia? ASAIO J 59: 69-74, 2013.

8. Fannelop T, Dahle GO, Salminen PR, Moen CA, Matre K, Mongstad A, Eliassen F, Segadal L and Grong K: Multidose cold oxygenated blood is superior to a single dose of Bretschneider HTK-cardioplegia in the pig. Ann Thorac Surg 87: 1205-1213, 2009.

9. Braathen B and Tønnessen T: Cold blood cardioplegia reduces the increase in cardiac enzyme levels compared with cold crystalloid cardioplegia in patients undergoing aortic valve replacement for isolated aortic stenosis. J Thorac Cardiovasc Surg 139: 874-880, 2010.

10. Hachida M, Nonoyama M, Bonkohara Y, Hanayama N, Saitou S, Maeda T, Ohkado A, Lu H and Koyanagi H: Clinical assessment of prolonged myocardial preservation for patients with a severely dilated heart. Ann Thorac Surg 64: 59-63, 1997.

11. Takeuchi K, Akimoto H, Maida K, Munakata M, Fukui K, Daitoku K, Takaya S, Suzuki S, Tabayashi K and Tanaka S: Myocardial protection of the pressure overload hypertrophied heart in human cardiac surgery by acceleration of anaerobic glycolysis. J Cardiovasc Surg (Torino) 43: 37-41, 2002.

12. Saitoh Y, Hashimoto M, Ku K, Kin S, Nosaka S, Masumura S and Nakayama K: Heart preservation in HTK solution: Role of coronary vasculature in recovery of cardiac function. Ann Thorac Surg 69: 107-112, 2000

13. Yang Q, Zhang RZ, Yim AP and He GW: Histidine-tryptophanketoglutarate solution maximally preserves endothelium-derived hyperpolarization factor mediated function during heart preservation: Comparison with University of Wisconsin solution. J Heart Lung Transpl 26: 352-359, 2004.

14. Careaga G, Salazar D, Téllez S, Sánchez O, Borrayo G and Argüero R: Clinical impact of histidine-ketoglutarate- tryptophan (HTK) cardioplegic solution on the perioperative period in open heart surgery patients. Arch Med Res 32: 296-299, 2001.
15. Liu YH, Dhakal BP, Keesakul C, Kacmarek RM, Lewis GD and Jiang Y: Continuous non-invasive cardiac output monitoring during exercise: Validation of electrical cardiometry with Fick and thermodilution methods. Br J Anaesth 117: 129-131, 2016.

16. Taylor K, La Rotta G, McCrindle BW, Manlhiot C, Redington A and Holtby $\mathrm{H}$ : A comparison of cardiac output by thoracic impedance and direct fick in children with congenital heart disease undergoing diagnostic cardiac catheterization. J Cardiothorac Vasc Anesth 25: 776-779, 2011.

17. Nishimura K, Ban T, Soneda J, Hirata K, Hanada T, Motoi T, Takeuchi T and Joho K: Anatomic correction of complete transposition of the great arteries by Lecompte procedure. Kyobu Geka 37: 303-307, 1984 (In Japanese).

18. Richard DW: Blood or crystalloid cardioplegia: Which is better? Eur J Cardiothorac Surg 43: 532-533, 2013.

19. Bojan M, Peperstraete H, Lilot M, Tourneur L, Vouhé P and Pouard P: Cold histidine-tryptophan-ketoglutarate solution and repeated oxygenated warm blood cardioplegia in neonates with arterial switch operation. Ann Thorac Surg 95: 1390-1396, 2013.

20. Gates RN, Palafox BA and Parker B: Results with all blood retrograde microplegia as a myocardial protection strategy for complex neonatal arch reconstruction. ASAIO J 54: 451-453, 2008.

21. Pouard P, Mauriat P, Ek F, Haydar A, Gioanni S, Laquay N, Vaccaroni L and Vouhé PR: Normothermic cardiopulmonary bypass and myocardial cardioplegia protection for neonatal arterial switch operation. Euro J Cardio-Thorac Surg 30: 695-699, 2006.

22. Korun O, Özkan M, Terzi A, Așkın G, Sezgin A and Aşlamacı S: The comparison of the effects of Bretschneider's histidine-tryptophan-ketoglutarate and conventional crystalloid cardioplegia on pediatric myocardium at tissue level. Artif Organs 37: 76-81, 2013.

23. Liu YL, Hu SS, Shen XD, Li SJ, Wang X, Yan J, Wu X, Huang JB and Kong B: Midterm results of arterial switch operation in older patients with severe pulmonary hypertension. Ann Thorac Surg 90: 848-855, 2010.

24. Huang JB, Liu YL, Sun PW, Lv XD, Bo K and Fan XM: Novel strategy for treatment of pulmonary arterial hypertension: Enhancement of apoptosis. Lung 188: 179-189, 2010.

25. Huang JB, Liang J, Zhao XF, Wu WS and Zhang F: Epigenetics: Novel mechanism of pulmonary hypertension. Lung 191: 601-610, 2013.

26. Kim JT, Park YH, Chang YE, Byon HJ, Kim HS, Kim CS, Lim HG, Kim WH, Lee JR and Kim YJ: The effect of cardioplegic solution-induced sodium concentration fluctuation on postoperative seizure in pediatric cardiac patients. Ann Thorac Surg 91: 1943-1948, 2011.

27. O'Brien JD, Howlett SE, Burton HJ, O'Blenes SB, Litz DS and Friesen CL: Pediatric cardioplegia strategy results in enhanced calcium metabolism and lower serum troponin T. Ann Thorac Surg 87: 1517-1523, 2009.

28. Lueck S, Preusse CJ and Welz A: Clinical relevance of HTK-induced hyponatremia. Ann Thorac Surg 95: 1844-1855, 2013. 